\title{
Analysis of liver steatosis analysis and controlled attenuation parameter for grading liver steatosis in patients with chronic hepatitis B
}

\author{
Xinping Ren ${ }^{1,2 \#}$, Shujun Xia ${ }^{1 \#}$, Lu Zhang ${ }^{1}$, Ruokun Li $^{3}$, Wei Zhou ${ }^{1}$, Ri Ji ${ }^{1}$, Jianqiao Zhou ${ }^{1}$, Jingyan Tian ${ }^{4}$, \\ Weiwei Zhan ${ }^{1}$ \\ ${ }^{1}$ Department of Ultrasound, Ruijin Hospital, School of Medicine, Shanghai Jiaotong University, Shanghai, China; ${ }^{2}$ Department of Ultrasound, Wuxi \\ Branch of Ruijin Hospital, Wuxi, China; ${ }^{3}$ Institute of Endocrinology, School of Medicine, Shanghai Jiaotong University, Shanghai, China; ${ }^{4}$ Institute \\ of Endocrine and Metabolic Diseases, Department of Endocrinology and Metabolism, Ruijin Hospital, School of Medicine, Shanghai Jiaotong \\ University, Shanghai, China
}

\#These authors contributed equally to this work.

Correspondence to: Xinping Ren; Weiwei Zhan. Department of Ultrasound, Ruijin Hospital, Shanghai Jiaotong University School of Medicine, No. 197, Ruijin 2 Road, Shanghai 200025, China. Email: peaceheart80@163.com; shanghairuijin@126.com.

\begin{abstract}
Background: Chronic hepatitis B is the most common chronic liver disease in China. For patients with chronic hepatitis B, steatosis increases the risk of cirrhosis and hepatocellular carcinoma. This study aimed to analyze and compare the clinical value of a newly developed ultrasound attenuation parameter, liver steatosis analysis (LiSA), acquired by Hepatus (Mindray, China), and controlled attenuation parameter (CAP), a widely used ultrasound attenuation parameter acquired by FibroScan (Echosens, France), for grading liver steatosis in patients with chronic hepatitis B infection.
\end{abstract}

Methods: A total of 203 patients were divided into two groups according to liver fat content validated by liver biopsy: group 1 (liver fat content $<10 \%$ ) and group 2 (liver fat content $\geq 10 \%$ ). All patients underwent LiSA and CAP examinations. Receiver operating characteristic (ROC) curves were calculated for the two ultrasound attenuation tools.

Results: Both LiSA and CAP successfully discriminated between patients in group 1 and group 2 . ROC curves showed that both tools had good diagnostic ability (AUC: $>0.7$ ) for steatosis $\geq 10 \%$, and the performance of LiSA was significantly better than CAP (AUC: 0.859 vs. 0.801, P=0.048). Using optimal cut-off points, LiSA had specificity and sensitivity of $96.23 \%$ and $76.08 \%$, respectively, for the diagnosis of steatosis $\geq 10 \%$, compared to $91.53 \%$ and $72.10 \%$, respectively, for CAP.

Conclusions: LiSA and CAP are extremely efficient tools for assessing liver steatosis, even at a low grade. Both parameters are non-invasive, inexpensive, and easy to use, and can provide immediate results with high sensitivity.

Keywords: Liver steatosis; chronic hepatitis B; ultrasound attenuation parameter; liver steatosis analysis (LiSA); controlled attenuation parameter (CAP)

Submitted Dec 24, 2019. Accepted for publication Sep 30, 2020.

doi: $10.21037 /$ qims-19-1091

View this article at: http://dx.doi.org/10.21037/qims-19-1091

\section{Introduction}

Liver steatosis is defined by the accumulation of lipid droplets, primarily triglycerides, in hepatocytes $(1,2)$.
Steatosis has become a common finding of liver biopsies as obesity and metabolic syndrome have risen in prevalence worldwide, and it may co-exist with other chronic liver diseases, such as chronic hepatitis B infection $(3,4)$. 
Several noninvasive techniques can be used to diagnose liver steatosis, including ultrasound, computed tomography (CT), and magnetic resonance imaging (MRI). Conventional $\mathrm{B}$-mode ultrasonography is the most widely used technique to detect the presence of liver steatosis; however, it is not completely sensitive, especially when there is less than $30 \%$ hepatic fat infiltration $(5,6)$. CT also has limited sensitivity for quantifying liver steatosis; moreover, this technique involves radiation exposure (7). MRI, particularly proton MRI spectroscopy, is the most reliable method for the quantification of fatty liver disease; however, the examination and the image-processing procedures tend to be complex, and the high cost restricts this method's regular use (8).

Recently, controlled attenuation parameter (CAP), a physical parameter acquired with the FibroScan (Echosens, France), has been widely used to assess steatosis $(9,10)$. FibroScan is an ultrasound-based vibration-controlled transient elastography (VCTE) device that is used to detect hepatic fibrosis-associated liver elasticity. CAP, which is based on FibroScan, measures the ultrasound attenuation at the center frequency of the $\mathrm{M}$ probe $(3.5 \mathrm{MHz})$. CAP is reported to have an excellent performance in the diagnosis of more than $10 \%$ of liver steatosis cases $(11,12)$. However, because CAP is not guided by two-dimensional gray-scale sonography, it cannot effectively avoid the biliary tract, large blood vessels, or intrahepatic masses. In practical operations, CAP is often timeconsuming, difficult to use, and even fails in the detection of liver steatosis $(13,14)$. Liver steatosis analysis (LiSA) is a newly developed ultrasound attenuation parameter for the diagnosis of hepatic steatosis that is based on a visual quantitative measuring platform, Hepatus (Mindray, China).

Chronic hepatitis B is the most common chronic liver disease in China. For chronic hepatitis B patients with steatosis, the risks of cirrhosis and hepatocellular carcinoma are increased $(15,16)$. It is important to screen for steatosis in patients with chronic hepatitis B. Therefore, we selected patients with $\mathrm{HBV}$ infection as our study subjects. This study aimed to evaluate the clinical value of LiSA in differentiating significant $(\geq 10 \%)$ from insignificant $(<10 \%)$ steatosis in patients with chronic hepatitis B, and to compare its clinical value with that of CAP.

\section{Methods}

\section{Patients}

All patients with HBV-related chronic liver disease who underwent percutaneous liver biopsy in the ultrasound unit of the Department of Infectious Disease in Ruijin Hospital between November 2018 and October 2019 were screened for inclusion. The exclusion criteria were as follows: (I) patients with regular alcohol consumption in the past 12 months, which was defined as more than 140 grams per week for men or more than 70 grams per week for women; (II) patients with other liver diseases that could cause steatosis, such as an infection or combined infection with other hepatitis virus, drug-induced liver injury, Wilson's disease, autoimmune hepatitis; (III) patients with total parenteral nutrition, liver transplantation patients, other end-stage medical diseases, or malignant tumors; (IV) patients with an aspartate aminotransferase (AST) or alanine aminotransferase (ALT) level $>5$ times the upper limit of normal values; (V) patients with a skin-toliver capsule distance of $>25 \mathrm{~mm}$, or a body mass index (BMI) of $\geq 28 \mathrm{~kg} / \mathrm{m}^{2}$.

For all enrolled patients, blood samples, liver stiffness measurement, CAP and LiSA assessment, and liver biopsy were performed on the same day. The following information was also collected at the time of liver steatosis evaluation: weight, height, waist circumference, arterial hypertension, diabetes, and alcohol and tobacco use.

\section{Ethical statement and written informed consent}

The study was approved by the ethics committee of Ruijin Hospital (No. 2017-72), and written informed consent was obtained from each patient. The patients did not receive any financial compensation.

\section{Equipment}

Measurement of LiSA was performed with Hepatus, a newly developed ultrasound machine, with a $3.5-\mathrm{MHz}$ phased array probe (LFP5-1U, Mindray, Re 6S, China). FibroScan with an $M$ probe (Echosens, FibroScan 502, France) was used to measure CAP. The attenuation of ultrasound propagation in the liver tissue is increased by fat accumulation. Therefore, the detection of ultrasonic attenuation coefficient is a quantitative method for evaluating the degree of liver steatosis (10). Both LiSA and CAP measure the total ultrasonic attenuation (go-and-return path) in the liver at $3.5 \mathrm{MHz}$, which is expressed in decibels per meter $(\mathrm{dB} / \mathrm{m})$. CAP measures the ultrasonic attenuation in the liver tissue along the vertical axis of the probe (one dimension), while LiSA detects the mean ultrasonic attenuations in liver tissue at the region of interest (two 
A

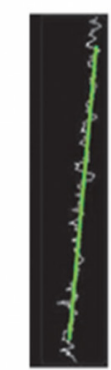

1D Calculation

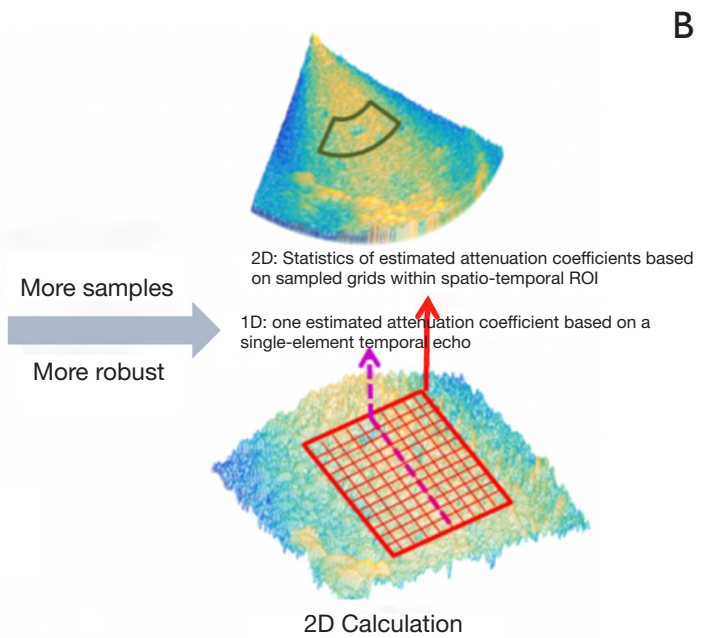

B

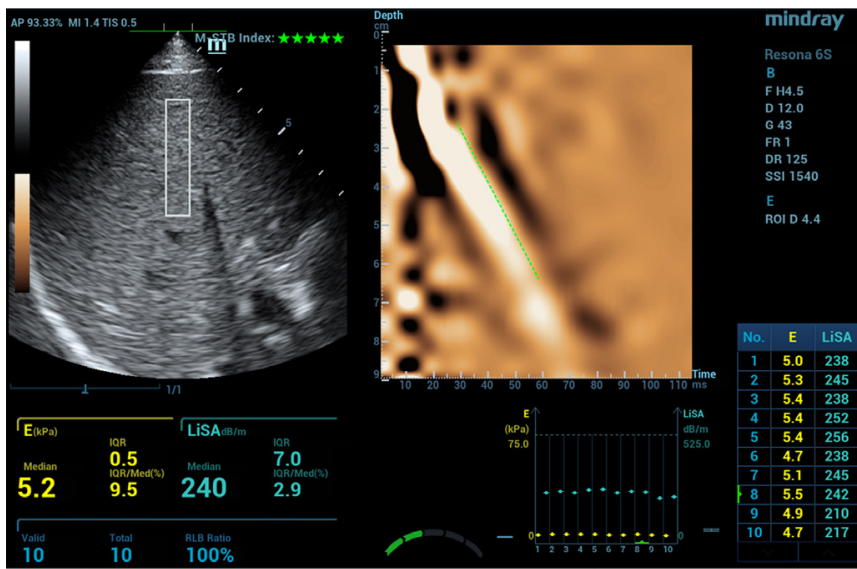

Figure 1 Liver steatosis analysis (LiSA). (A) The difference between controlled attenuation parameter (CAP) and LiSA in the detection of ultrasound attenuation in the liver. (B) Actual measurement screen of LiSA.

dimension) (Figure 1A). The actual visual measurement screen of LiSA is shown in Figure $1 B$.

\section{Measurement of liver steatosis}

Experienced operators performed all CAP and LiSA examinations according to the manufacturer's instructions. During the examinations, all patients were in the dorsal decubitus position, with their right arm extended and lifted. Imaging was used to assess the right lobe of the liver through the intercostal approach with a calm breath hold of 6-7 seconds. Detection with successful measurements in fewer than 8 in 10 attempts was deemed a failure. The success rate was calculated as successful examinations versus total examinations. The final LiSA or CAP value was the median of individual measurements, and the interquartile range (IQR) $<40 \mathrm{~dB} / \mathrm{m}$ was used as a quality criterion.

\section{Laboratory examinations}

The blood biochemical parameters of the patients were tested including fasting glucose, liver function [ALT, AST, $\gamma$-glutamyltransferase $(\gamma-\mathrm{GT})$, and total bilirubin (TB)], and lipids [total cholesterol (TC), triglyceride (TG), high-density lipoprotein cholesterol (HDL-C), and lowdensity lipoprotein cholesterol (LDL-C)]. All biochemical parameters were detected by standard automated laboratory methods using commercially available kits in accordance with the manufacturers' protocols.

\section{Liver bistology}

Liver biopsy was performed on the same day as ultrasound measurement and was conducted by senior operators using an automated biopsy gun with a $16 \mathrm{G}$ needle (Bard Magnum, USA). Samples were required to be $>1.5 \mathrm{~cm}$ in length and contain $>10$ portal tracts. The samples of damaged liver tissue were not included in this study. The specimens were fixed in formalin, embedded in paraffin, and continuously sectioned. Then, the samples were stained with hematoxylin and eosin, and Masson's trichromic stain to detect collagen. Patients were categorized by visual assessment as group 1: steatosis $<10 \%$ of hepatocytes, and group 2: steatosis $\geq 10 \%$. Hepatic fibrosis stage and inflammatory activity grade were evaluated according to the Scheuer scoring system. The final pathological report was made by two senior pathologists, who were blinded to the results of LiSA, CAP, and liver stiffness measurement.

\section{Statistical analysis}

Continuous variables were expressed as mean \pm standard deviation (s.d.) and were compared using the Student's t test or Mann-Whitney $U$ test. Categorical variables were compared using the $\chi^{2}$ test. Relationships between LiSA or CAP and clinical parameters were evaluated using Pearson's correlation coefficient. Factors associated with steatosis $>10 \%$ on liver biopsy were analyzed by logistic regression. Receiver operating characteristic (ROC) curves were constructed and the area under the ROC curves 
Table 1 Characteristics of the study participants

\begin{tabular}{|c|c|c|c|}
\hline Characteristics & Group $1(n=168)$ & Group $2(n=35)$ & $P$ value \\
\hline Sex (female/male) & $13 / 54$ & $10 / 29$ & 0.098 \\
\hline $\mathrm{BMI}\left(\mathrm{kg} / \mathrm{m}^{2}\right)$ & $21.05 \pm 3.90$ & $22.34 \pm 2.73$ & 0.078 \\
\hline Waist circumference $(\mathrm{cm})$ & $79.96 \pm 13.21$ & $87.81 \pm 11.53$ & 0.091 \\
\hline ALT (IU/L) & $31.06 \pm 12.19$ & $40.01 \pm 22.05$ & 0.182 \\
\hline AST (IU/L) & $41.18 \pm 11.25$ & $40.97 \pm 12.19$ & 0.126 \\
\hline$\gamma$-GT (IU/L) & $31.05 \pm 11.98$ & $35.78 \pm 21.12$ & 0.056 \\
\hline Total bilirubin ( $\mu \mathrm{mol} / \mathrm{L})$ & $10.86 \pm 12.35$ & $11.78 \pm 12.12$ & 0.067 \\
\hline HDL-C (mmol/L) & $0.96 \pm 0.38$ & $1.20 \pm 0.64$ & 0.071 \\
\hline LDL-C (mmol/L) & $2.82 \pm 0.86$ & $2.23 \pm 0.78$ & 0.067 \\
\hline Fibrosis grade & & & 0.052 \\
\hline$\geq 2$ & 18 & 13 & \\
\hline$<2$ & 150 & 22 & \\
\hline Inflammation grade & & & 0.059 \\
\hline$\geq 2$ & 7 & 6 & \\
\hline$<2$ & 162 & 29 & \\
\hline
\end{tabular}

BMI, body mass index; ALT, alanine aminotransferase; AST, aspartate aminotransferase; $\gamma$-GT, $\gamma$-glutamyltransferase; TC, total cholesterol; TG, triglyceride; HDL-C, high-density lipoprotein cholesterol; LDL-C, low-density lipoprotein cholesterol.

(AUCs) were calculated for LiSA and CAP. The Youden index was used to determine the diagnostic threshold and corresponding diagnostic sensitivity and specificity. The AUC values were compared using the DeLong test. Statistical analyses were performed with SPSS software (version 22.0; IBM Corp, Armonk, NY, USA). $\mathrm{P}<0.05$ was considered as statistically significant.

\section{Results}

\section{Clinical and histological characteristics of patients}

A total of 203 patients who met the inclusion criteria were included in this study. The patients were divided into group 1 (liver fat content $<10 \%$ ) or group 2 (liver fat content $\geq 10 \%$ ) according to liver fat content validated by liver biopsy. The clinical and histological characteristics of the two groups of patients are listed in Table 1. All clinical and histological indicators measured in this study failed to discriminate the two groups of patients.

\section{Performances of LiSA and CAP}

The success rates of LiSA and CAP for the measurement of liver steatosis were 100\% (203/203) and 98.52\% (200/203), respectively $(\mathrm{P}>0.05)$. The values of CAP in patients with $<10 \%$ and $\geq 10 \%$ steatosis were $211 \pm 46$ and $243 \pm 36 \mathrm{~dB} / \mathrm{m}$, respectively, and the values of LiSA in these patients were $207 \pm 41$ and $244 \pm 38 \mathrm{~dB} / \mathrm{m}$, respectively. Patients with severe steatosis had significantly higher CAP or LiSA values than those with less severe steatosis $(\mathrm{P}=0.024$ for CAP; $\mathrm{P}=0.032$ for LiSA) (Figure 2A). The ROC curves also indicated that both methods showed good diagnostic ability (AUC $>0.7$ ) for steatosis $\geq 10 \%$, and the performance of LiSA was significantly better than CAP (AUC 0.859 vs. 0.801, $\mathrm{P}=0.048)$. Using optimal cut-off points, LiSA 

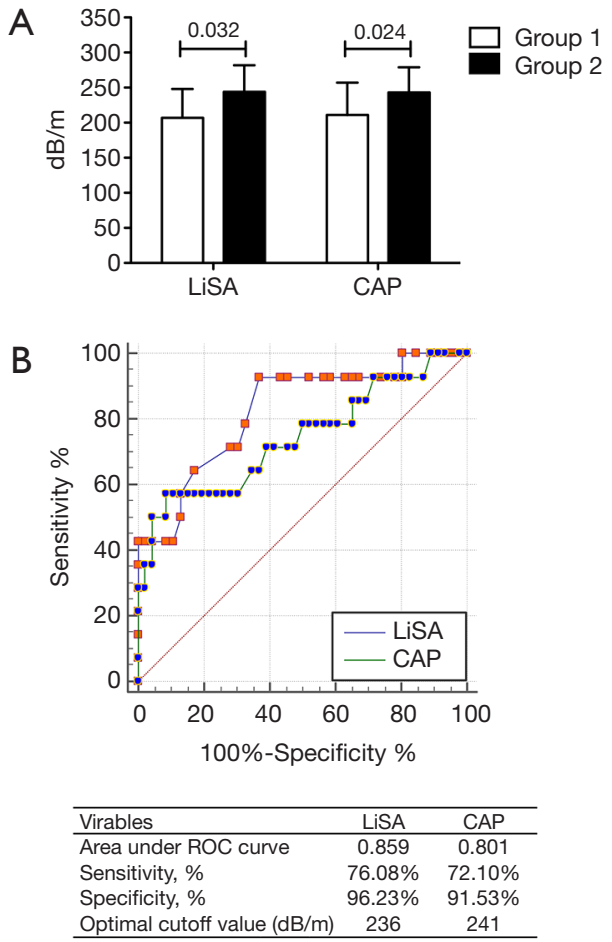

Figure 2 The performances of the two attenuation parameters for grading hepatic steatosis. (A) The values of liver steatosis determined using the two methods. (B) Receiver operating characteristic (ROC) curve analysis of the two methods. had a specificity and sensitivity of $96.23 \%$ and $76.08 \%$, respectively, for the diagnosis of steatosis $\geq 10 \%$, compared to $91.53 \%$ and $72.10 \%$, respectively, for CAP (Figure $2 B$ ).

Both LiSA and CAP were significantly correlated with BMI (LiSA: $r=0.769, \mathrm{P}=0.039$; CAP: $\mathrm{r}=0.716, \mathrm{P}=0.048$ ), fasting glucose (LiSA: $r=0.156, \mathrm{P}=0.041$; CAP: $r=0.167$, $\mathrm{P}=0.045$ ), and liver stiffness (LiSA: $\mathrm{r}=0.802, \mathrm{P}<0.001$; CAP: $\mathrm{r}=0.781, \mathrm{P}<0.001)$, but not with age, waist circumference, ALT, AST, $\gamma-\mathrm{GT}$, total bilirubin, TC, TG, HDL-C, or LDL-C (Table 2).

In univariate analysis, the factors associated with steatosis $\geq 10 \%$ on liver biopsy were BMI, waist circumference, TC, TG, HDL-C, CAP, and LiSA. In multivariate analysis, BMI (OR: 4.92, 95\% CI: 2.89-8.27, $\mathrm{P}<0.001$ ), waist circumference (OR: 2.01, 95\% CI: 1.09-3.02, $\mathrm{P}=0.037$ ), CAP (OR: 5.04, 95\% CI: $2.43-8.50, \mathrm{P}<0.001$ ), and LiSA (OR: 5.82, 95\% CI: 2.76-11.81, P<0.001) were independently associated with steatosis $\geq 10 \%$ on liver biopsy (Table 3).

\section{Discussion}

Liver steatosis is a common histological feature among patients with chronic liver diseases, and its coexistence can accelerate the progression of fibrosis and reduce the

Table 2 Factors associated with liver steatosis analysis (LiSA) and controlled attenuation parameter (CAP)

\begin{tabular}{lccccc}
\hline \multirow{2}{*}{ Variable } & \multicolumn{2}{c}{ LiSA } & & \multicolumn{2}{c}{ CAP } \\
\cline { 2 - 3 } \cline { 4 - 5 } Age & $r$ & $\mathrm{P}$ & $\mathrm{r}$ & $\mathrm{P}$ \\
BMI & 0.167 & 0.090 & 0.156 & 0.104 \\
Waist circumference & 0.769 & 0.039 & 0.716 & 0.048 \\
Fasting glucose & 0.517 & 0.085 & 0.509 & 0.079 \\
ALT & 0.156 & 0.041 & 0.167 & 0.045 \\
AST & -0.051 & 0.278 & -0.056 & 0.215 \\
$\gamma$-GT & -0.781 & 0.396 & -0.871 & 0.354 \\
Total bilirubin & 0.024 & 0.116 & 0.012 & 0.101 \\
TC & 0.011 & 0.102 & 0.091 & 0.118 \\
TG & 0.179 & 0.105 & 0.189 & 0.117 \\
HDL-C & 0.102 & 0.053 & 0.119 & 0.067 \\
LDL-C & -0.149 & 0.203 & -0.167 & 0.242 \\
Liver stiffness & 0.326 & 0.054 & 0.419 & 0.056 \\
\hline
\end{tabular}

BMI, body mass index; ALT, alanine aminotransferase; AST, aspartate aminotransferase; $\gamma$-GT, $\gamma$-glutamyltransferase; TC, total cholesterol; TG, triglyceride; HDL-C, high-density lipoprotein cholesterol; LDL-C, low-density lipoprotein cholesterol. 
Table 3 Factors associated with steatosis $\geq 10 \%$ on liver biopsy

\begin{tabular}{lccc}
\hline Variable & OR & $95 \% \mathrm{Cl}$ & $\mathrm{P}$ \\
\hline Univariate analysis & & & \\
BMI & 5.33 & $2.24-8.41$ & $<0.001$ \\
Waist circumference & 3.27 & $1.01-4.04$ & $<0.001$ \\
TC & 1.17 & $1.09-1.19$ & 0.005 \\
TG & 1.02 & $1.00-1.06$ & $<0.001$ \\
HDL-C & 0.49 & $0.37-0.61$ & 0.003 \\
CAP & 7.51 & $1.49-11.52$ & $<0.001$ \\
LiSA & 8.12 & $3.65-10.89$ & $<0.001$ \\
Multivariate analysis & & & \\
BMI & 4.92 & $2.89-8.27$ & $<0.001$ \\
Waist circumference & 2.01 & $1.09-3.02$ & 0.037 \\
CAP & 5.04 & $2.43-8.50$ & $<0.001$ \\
LiSA & 5.82 & $2.76-11.81$ & $<0.001$ \\
\hline
\end{tabular}

BMI, body mass index; TC, total cholesterol; TG, triglyceride; HDL-C, high-density lipoprotein cholesterol; CAP, controlled attenuation parameter; LiSA, liver steatosis analysis.

treatment response. Therefore, the ability to accurately diagnose liver steatosis is important for clinical decisionmaking and estimating prognosis. Biopsy still remains the gold standard for liver steatosis diagnosis and grading, although it has clear limitations. These include invasiveness, potential sampling error, and intra/inter observer variability $(17,18)$. Liver steatosis can also be diagnosed through noninvasive techniques, including ultrasound, CT, and MRI. However, unfortunately, conventional B-mode ultrasonography and CT can only be used to detect steatosis starting from around $30 \%$ of hepatocytes. Additional limitations include its operator dependence, high cost, and restricted availability (6-8).

New tools are needed to overcome these limitations. Fat accumulation will increase the attenuation of ultrasound propagation in the liver tissue; therefore, new tools based on ultrasound attenuation measurements obtained using an ultrasound scanner have been developed to evaluate hepatic steatosis. CAP of FibroScan is a practical example that is reported to show a high correlation with steatosis grades and to be able to evaluate steatosis efficiently (19). However, since FibroScan is not an imaging modality, CAP should be used in combination with a general purpose ultrasonic diagnostic system. To overcome the limitations of CAP, there are several tools that combine the echo attenuation measurement function with an ultrasonic diagnostic system for the accurate diagnosis of liver steatosis, including ultrasound-guided attenuation parameter (UGAP), attenuation imaging (ATI) modality, and attenuation coefficient (ATT) modality (20-22). UGAP was performed on LOGIQ E9 XDclear 2.0 ultrasound scanner (GE Healthcare, USA) with a C1-6-D phased array probe (frequency: $4 \mathrm{MHz}$ ) (20). The AUROCs of UGAP for the prediction of grade 1 (5-33\%), 2 (33-66\%), and 3 (>66\%) steatosis were $0.900,0.950$, and 0.959 , respectively. Contrastingly, in the same studied subjects, the AUROCs of CAP for the diagnosis of grade 1, 2, and 3 steatosis were $0.829,0.841$, and 0.817 , respectively. The AUROCs were significantly higher for UGAP than for CAP in the prediction of grade 2 and 3 steatosis (20). The ATI modality was installed on the Aplio i-series system with a convex probe (Canon Medical Systems Corporation, Japan) (21). The AUROCs of ATI for the diagnosis of grade 1, 2, and 3 steatosis were $0.85,0.91$, and 0.91 , respectively (21). ATT was performed using ultrasound (HI VISION Ascendus, Japan) and an EUP-C715 convex type probe (5-1 MHz; Hitachi, Japan) (22). The AUROCs of ATT for the diagnosis of grade 1, 2, and 3 steatosis were $0.79,0.87$, and 0.96 , respectively (22). Generally, these tools were found to have good diagnostic ability for hepatic steatosis, even for mild cases $(>5 \%)$.

In this study, the clinical value of LiSA, a newly developed ultrasound attenuation parameter acquired by Hepatus (Mindray, China), for the differentiation of significant $(\geq 10 \%)$ from insignificant steatosis $(<10 \%)$ was evaluated in patients with chronic hepatitis $\mathrm{B}$, and compared with that of CAP, the widely used ultrasound attenuation parameter acquired by FibroScan (Echosens, France). The use of the Hepatus system for the assessment of steatosis has not been reported. The ROC curves indicated that both LiSA and CAP had good diagnostic ability (AUC $>0.7$ ) for steatosis $\geq 10 \%$, and the performance of LiSA was significantly better than that of CAP (AUC 0.859 vs. 0.801). Using optimal cut-off points, LiSA also had higher specificity and sensitivity than CAP. Therefore, the findings of our study combined with those of other reports show that tools that combine ultrasound attenuation measurements with general ultrasonography seem to have an advantage over CAP, which has insufficient visual guidance.

The advantage of LiSA over CAP may be attributable to the following reasons. First, LiSA has a real-time image guidance system that can help operators to quickly find the effective detection windows and effectively avoid 
large blood vessels, biliary tract, and intrahepatic masses. Furthermore, CAP based on the FibroScan platform evaluates fat content through the detection of the average attenuation of ultrasonic signal generated by external lowfrequency vibration instrument in one-dimensional linear echo. It is a single channel signal system but consists of multiple signals. Meanwhile, LiSA, based on the Hepatus platform, assesses fat content by measuring the average attenuation of ultrasonic signals in the region of interest. The multi-element probe it uses can distinguish the details of different local positions in a certain range of space with higher resolution and more information. Therefore, the area of liver tissue detected by LiSA is much larger than that measured by CAP. However, further research should be conducted to test the effectiveness of this tool in larger study populations.

The limitations of this study included its small sample size, single-center nature, and focus on a single type of liver disease resulting in steatosis. Although, we used liver biopsy, which is the gold standard for the diagnosis of liver steatosis, as the diagnostic reference, the area detected by biopsy was smaller than that measured by LiSA and CAP, and might not represent the whole disease status of the liver. Future studies with a large sample size and patients from multiple medical centers should be carried out.

\section{Conclusions}

LiSA is a non-invasive, inexpensive tool for diagnosing and grading steatosis. It can be easily applied to obtain immediate results with high sensitivity.

\section{Acknowledgments}

Funding: None.

\section{Footnote}

Conflicts of Interest: All authors have completed the ICMJE uniform disclosure form (available at http://dx.doi. org/10.21037/qims-19-1091). The authors have no conflicts of interest to declare.

Ethical Statement: The study was approved by the ethics committee of Ruijin Hospital (No. 2017-72) and written informed consent was obtained from all patients.

Open Access Statement: This is an Open Access article distributed in accordance with the Creative Commons Attribution-NonCommercial-NoDerivs 4.0 International License (CC BY-NC-ND 4.0), which permits the noncommercial replication and distribution of the article with the strict proviso that no changes or edits are made and the original work is properly cited (including links to both the formal publication through the relevant DOI and the license). See: https://creativecommons.org/licenses/by-nc-nd/4.0/.

\section{References}

1. Browning JD, Szczepaniak LS, Dobbins R, Nuremberg P, Horton JD, Cohen JC, Cohen JC, Grundy SM, Hobbs

$\mathrm{HH}$. Prevalence of hepatic steatosis in an urban population in the United States: impact of ethnicity. Hepatology 2004;40:1387-95.

2. Hu F, Yang R, Huang Z, Wang M, Yuan F, Xia C, Wei Y, Song B. 3D Multi-Echo Dixon technique for simultaneous assessment of liver steatosis and iron overload in patients with chronic liver diseases: a feasibility study. Quant Imaging Med Surg 2019;9:1014-24.

3. Machado MV, Oliveira AG, Cortez-Pinto H. Hepatic steatosis in hepatitis B virus infected patients: metaanalysis of risk factors and comparison with hepatitis $\mathrm{C}$ infected patients. J Gastroenterol Hepatol 2011;26:1361-7.

4. Wang CC, Hsu CS, Liu CJ, Kao JH, Chen DS. Association of chronic hepatitis $\mathrm{B}$ virus infection with insulin resistance and hepatic steatosis. J Gastroenterol Hepatol 2008;23:779-82.

5. Zhou Z, Zhang Q, Wu W, Lin YH, Tai DI, Tseng JH, Lin YR, Wu S, Tsui PH. Hepatic steatosis assessment using ultrasound homodyned-K parametric imaging: the effects of estimators. Quant Imaging Med Surg 2019;9:1932-47.

6. Hernaez R, Lazo M, Bonekamp S, Kamel IR, Brancati FL, Guallar E, Clark JM. Diagnostic accuracy and reliability of ultrasonography for the detection of fatty liver: A metaanalysis. Hepatology 2011;54:1082-90.

7. Bohte AE, van Werven JR, Bipat S, Stoker J. The diagnostic accuracy of US, CT, MRI and 1H-MRS for the evaluation of hepatic steatosis compared with liver biopsy: a meta-analysis. Eur Radiol 2011;21:87-97.

8. Noworolski SM, Lam MM, Merriman RB, Ferrell L, Qayyum A. Liver steatosis: concordance of MR imaging and MR spectroscopic data with histologic grade. Radiology 2012;264:88-96.

9. Sasso M, Tengher-Barna I, Ziol M, Miette V, Fournier C, Sandrin L, Poupon R, Cardoso AC, Marcellin P, Douvin C, Ledinghen V, Trinchet JC, Beaugrand M. 
Novel controlled attenuation parameter for noninvasive assessment of steatosis using Fibroscan(®): validation in chronic hepatitis C. J Viral Hepat 2012;19:244-53.

10. Sasso M, Miette V, Sandrin L, Beaugrand M. The controlled attenuation parameter (CAP): a novel tool for the non-invasive evaluation of steatosis using Fibroscan. Clin Res Hepatol Gastroenterol 2012;36:13-20.

11. Sasso M, Beaugrand M, de Ledinghen V, Douvin C, Marcellin P, Poupon R, Sandrin L, Miette V. Controlled attenuation parameter (CAP): a novel $\mathrm{VCTE}^{\mathrm{TM}}$ guided ultrasonic attenuation measurement for the evaluation of hepatic steatosis: preliminary study and validation in a cohort of patients with chronic liver disease from various causes. Ultrasound Med Biol 2010;36:1825-35.

12. de Lédinghen V, Vergniol J, Capdepont $M$, Chermak F, Hiriart JB, Cassinotto C, Merrouche W, Foucher J, Brigitte le B. Controlled attenuation parameter (CAP) for the diagnosis of steatosis: a prospective study of 5323 examinations. J Hepatol 2014;60:1026-31.

13. Friedrich-Rust M, Müller C, Winckler A, Kriener S, Herrmann E, Holtmeier J, Poynard T, Vogl TJ, Zeuzem S, Hammerstingl R, Sarrazin C. Assessment of liver fibrosis and steatosis in PBC with FibroScan, MRI, MRspectroscopy, and serum markers. J Clin Gastroenterol 2010;44:58-65.

14. Wilder J, Patel K. The clinical utility of FibroScan(®) as a noninvasive diagnostic test for liver disease. Med Devices (Auckl) 2014;7:107-14.

15. Cho H, Chang Y, Lee JH, Cho YY, Nam JY, Lee YB, Lee DH, Cho EJ, Yu SJ, Kim YJ, Lee JM, Yoon JH. Radiologic nonalcoholic fatty liver disease increases the risk of hepatocellular carcinoma in patients with suppressed chronic hepatitis B. J Clin Gastroenterol 2020;54:633-41.

16. Li Q, Huang C, Xu W, Hu Q, Chen L. Accuracy of FibroScan in analysis of liver fibrosis in patients with

Cite this article as: Ren X, Xia S, Zhang L, Li R, Zhou W, Ji R, Zhou J, Tian J, Zhan W. Analysis of liver steatosis analysis and controlled attenuation parameter for grading liver steatosis in patients with chronic hepatitis B. Quant Imaging Med Surg 2021;11(2):571-578. doi: 10.21037/qims-19-1091 concomitant chronic Hepatitis B and nonalcoholic fatty liver disease. Medicine (Baltimore) 2020;99:e20616.

17. Vuppalanchi R, Unalp A, Van Natta ML, Cummings OW, Sandrasegaran KE, Hameed T, Tonascia J, Chalasani N. Effects of liver biopsy sample length and number of readings on sampling variability in nonalcoholic Fatty liver disease. Clin Gastroenterol Hepatol 2009;7:481-6.

18. Regev A, Berho M, Jeffers LJ, Milikowski C, Molina EG, Pyrsopoulos NT, Feng ZZ, Reddy KR, Schiff ER. Sampling error and intraobserver variation in liver biopsy in patients with chronic HCV infection. Am J Gastroenterol 2002;97:2614-8.

19. Myers RP, Pollett A, Kirsch R, Pomier-Layrargues G, Beaton M, Levstik M, Duarte-Rojo A, Wong D, Crotty P, Elkashab M. Controlled Attenuation Parameter (CAP): a noninvasive method for the detection of hepatic steatosis based on transient elastography. Liver Int 2012;32:902-10.

20. Fujiwara Y, Kuroda H, Abe T, Ishida K, Oguri T, Noguchi S, Sugai T, Kamiyama N, Takikawa Y. The B-mode imageguided ultrasound attenuation parameter accurately detects hepatic steatosis in chronic liver disease. Ultrasound Med Biol 2018;44:2223-32.

21. Tada T, Iijima H, Kobayashi N, Yoshida M, Nishimura T, Kumada T, Kondo R, Yano H, Kage M, Nakano C, Aoki T, Aizawa N, Ikeda N, Takashima T, Yuri Y, Ishii N, Hasegawa K, Takata R, Yoh K, Sakai Y, Nishikawa H, Iwata Y, Enomoto H, Hirota S, Fujimoto J, Nishiguchi S. Usefulness of attenuation imaging with an ultrasound scanner for the evaluation of hepatic steatosis. Ultrasound Med Biol 2019;45:2679-87.

22. Tamaki N, Koizumi Y, Hirooka M, Yada N, Takada H, Nakashima O, Kudo M, Hiasa Y, Izumi N. Novel quantitative assessment system of liver steatosis using a newly developed attenuation measurement method. Hepatol Res 2018;48:821-8. 